\title{
Exact Ground State Energy of the Strong-Coupling Polaron ${ }^{\star}$
}

\author{
Elliott H. Lieb' ${ }^{1}$ Lawrence E. Thomas ${ }^{2}$ \\ ${ }^{1}$ Departments of Physics and Mathematics, Jadwin Hall, Princeton University, P. O. Box 708, Princeton, New \\ Jersey 08544, USA \\ 2 Department of Mathematics, University of Virginia, Charlottesville, Virginia 22903, USA
}

Received: 6 May 1996/ Accepted: 20 May 1996

Dedicated to the memory of R.L. Dobrushin and P.W. Kasteleyn, two founders of modern statistical mechanics

\begin{abstract}
The polaron has been of interest in condensed matter theory and field theory for about half a century, especially the limit of large coupling constant, $\alpha$. It was not until 1983, however, that a proof of the asymptotic formula for the ground state energy was finally given by using difficult arguments involving the large deviation theory of path integrals. Here we derive the same asymptotic result, $E_{0} \sim-C \alpha^{2}$, and with explicit error bounds, by simple, rigorous methods applied directly to the Hamiltonian. Our method is easily generalizable to other settings, e.g., the excitonic and magnetic polarons.
\end{abstract}

The polaron Hamiltonian of Fröhlich [1] is a model for the Coulomb interaction of one or more electrons with the quantized phonons of an ionic crystal. In the course of time it was also seen to be an interesting field theory model of non-relativistic particles interacting with a scalar boson field, and it was widely studied [2-9] in both contexts.

The model has one dimensionless coupling constant, $\alpha$, and it was noticed very early that there seems to be a qualitative difference in the ground state between the weak coupling regime, well described by perturbation theory [8], in which the electron is spread out, and the strong coupling regime in which the electron appears to be trapped in a phonon hole of its own making. (This question of trapping seems to have first been considered by Landau [10] in the context of a classical phonon field.)

By now it seems doubtful that such a trapping actually occurs [7, 11, 12], but it remains true that the calculation of the ground state energy, $E_{0}(\alpha)$, is very different in the two regimes. The strong coupling theory was studied by Pekar [2] (see also [3-5, 9]) who hypothesized that in this limit the total ground state wave function $\Psi$ could be taken to be a product of an electronic function $\phi(x)$ and a phonon function $|\xi\rangle$. It is a fact that this ansatz, called the adiabatic approximation, yields exactly the same result for the ground state energy as the corresponding model with classical phonons. This

\footnotetext{
* (c) 1996 retained by the authors. Faithful reproduction of this article, by any means, is permitted for non-commercial purposes.
} 
stems from the fact that the phonon operators enter only linearly and quadratically in the Hamiltonian.

Fröhlich's Hamiltonian in appropriate units is

$$
H=\mathbf{p}^{2}+\sum_{\mathbf{k}} a_{\mathbf{k}}^{*} a_{\mathbf{k}}+\left(\frac{4 \pi \alpha}{V}\right)^{1 / 2} \sum_{\mathbf{k}}\left[\frac{a_{\mathbf{k}}}{|\mathbf{k}|} e^{i \mathbf{k} \cdot \mathbf{x}}+\text { h.c. }\right],
$$

where $\mathbf{p}=-i \nabla$ is the electron momentum operator, $\mathbf{x}$ is its coordinate, and $V$ is the volume of the crystal, which tends to infinity. The k's are the usual normal modes (e.g., $\mathbf{k}=2 \pi V^{-1 / 3}\left(n_{1}, n_{2}, n_{3}\right)$ for a cubic box $)$ and, as usual, $V^{-1} \sum_{\mathbf{k}} \rightarrow(2 \pi)^{-3} \int d^{3} k$. Also, as usual, the $\mathbf{k}=0$ mode is omitted. When $\langle\Psi|H| \Psi\rangle$ is computed with Pekar's ansatz one easily finds that the phonon part $|\xi\rangle$ can be easily evaluated by "completing the square". and the resulting $\phi$ minimizes the energy

$$
\mathcal{E}(\phi) \equiv \int|\nabla \phi|^{2} d^{3} x-\alpha \iint \frac{\phi(\mathbf{x})^{2} \phi(\mathbf{y})^{2}}{|\mathbf{x}-\mathbf{y}|} d^{3} x d^{3} y
$$

subject to $\int \phi(x)^{2} d^{3} x=1$. This, as we mentioned above, is what one would get if the $a_{\mathbf{k}}$ 's are replaced by c-numbers $\xi_{\mathbf{k}}$.

It has been proved [13] that there is exactly one $\phi$, up to translations, that minimizes $\mathcal{E}(\phi)$. By scaling, the minimum energy (call it $E_{P}(\alpha)$ ) is proportional to $-\alpha^{2}$. The question we address is this: Is this energy, $E_{P}(\alpha)$, asymptotically exact as $\alpha$ tends to infinity? Since it is given by a variational calculation, $E_{P}(\alpha)$ is an upper bound to $E_{0}(\alpha)$; the problem is to find a lower bound that agrees with $E_{P}(\alpha)$ to leading order. We provide such a lower bound in (31) below.

For a long time more or less the only thing that could be done to validate $E_{P}(\alpha)$ rigorously was a lower bound [14] of the right order, $-\alpha^{2}$, but which was about a factor of 3 too large. It was not until 1983, with a precursor in [15], that a complete proof of the asymptotic correctness of $E_{P}(\alpha)$ was given by Donsker and Varadhan [16]. The proof starts with a familiar expression for the ground state energy,

$$
E_{0}(\alpha)=\lim _{\beta \rightarrow \infty} \frac{1}{\beta} \ln \left\langle\Psi\left|e^{-\beta H}\right| \Psi\right\rangle,
$$

with $\Psi$ such that its spectral resolution contains the ground state energy or low energy spectrum of $H$ but is otherwise arbitrary. Now in fact there is a relatively simple Feynman-Kac formula for the semigroup $e^{-\beta H}$ first noted by Feynman in [18]; by integrating out the phonon coordinates in this formula, one finds that the right side of (3) can be replaced by the expression

$$
\lim _{\beta \rightarrow \infty} \frac{1}{\beta} \ln E\left\{\exp \left(\alpha \int_{0}^{\beta} \int_{0}^{s} \frac{e^{-(t-s)}}{|\mathbf{x}(t)-\mathbf{x}(s)|} d s d t\right)\right\} .
$$

Here, $E$ represents path space expectation for the Brownian motion $\mathbf{x}(t)$. The FeynmanKac formula and functional integral are discussed in some detail by Ginibre [19] and Roepstorff [20].

It is this limit (4) that Donsker and Varadhan analyze, in effect showing that the Pekar functional (2) provides a kind of large deviation rate function for the functional integral in (4). Their proof is very far from simple and requires an enormous knowledge of large deviation theory of Brownian motion, a subject unknown to most physicists, and it does not generalize easily to other models. Owing to the double integral in (4) 
it is necessary to construct a large deviation theory for "infinitely many variables". Moreover, no error bounds were provided [17]. We note that previously Feynman had used this formula to estimate the ground state energy from above, but his estimate is not sharp even for $\alpha \rightarrow \infty$. See also [21] where this functional integral is again employed to obtain a lower bound on the ground state energy which, however, is not sharp.

Regarding the matter of error bounds, we note that Gross [5] takes advantage of momentum conservation to eliminate three phonon degrees of freedom and transforms the Hamiltonian $H$ to a new form involving a sum of $\alpha^{2}-, \alpha^{0}$ - and $\alpha^{-2}$-terms. (He continues to employ the Pekar ansatz.) This form of the Hamiltonian perhaps suggests that the ground state energy itself has an asymptotic expansion in inverse powers of $\alpha^{2}$. But the terms in this transformed Hamiltonian are unbounded operators, and so the order of their contributions to the energy is by no means obvious. The bound we obtain on the error is actually $O\left(\alpha^{9 / 5}\right)$, which is to be compared with the main term $O\left(\alpha^{2}\right)$.

In any event, there is the following problem: The Pekar ansatz is based on the physically appealing notion that at large coupling the phonons cannot follow the rapidly moving electron (as they do at weak coupling) and so resign themselves to interacting only with the "mean" electronic density, $\phi(x)^{2}$. What, exactly, do these somewhat anthropomorphic words mean? If they are so very physical, they should be quantifiable and it should not take four decades to build a proof of their correctness. Moreover, one should be able to find a proof that is relatively simple (since the idea is a simple one), and one that also yields some kind of quantitative error estimate. It should also be robust enough to allow an easy extension to some variations of $H$, such as having several electrons instead of one (the polaronic exciton), inclusion of magnetic fields, an electron kinetic energy, $T(\mathbf{p})$, other than $\mathbf{p}^{2}$ (for which the semigroup exp $[-T(\mathbf{p})]$ might not even have a positive kernel), accommodation of $\mathbf{k}$-dependence and nonlinearity in the phonon self energies, electron-phonon interaction energies other than $1 /|\mathbf{k}|$, etc.

The method presented here satisfies, we believe, the criteria of simplicity, robustness and, of course, rigor. For this reason it might be of general interest in condensed matter theory or field theory. Despite its generality we shall, for clarity, restrict our discussion to the original Hamiltonian, $H$.

Before going into the details, it is helpful to give an overview of our method. The first thing to notice is that Pekar's ansatz amounts, mathematically, to saying that we can replace the operators $a_{\mathbf{k}}$ by c-numbers $\xi_{\mathbf{k}}$. One might think, at first, that such a replacement always leads to a lower bound for the energy, but this is false. (If it were true, the small $\alpha$ energy would have to be proportional to $-\alpha^{2}$ instead of the much more negative, correct value $-\alpha$.) To pursue this idea, nevertheless, the natural tool to think of is coherent states for each phonon mode $\mathbf{k}$. As is well known, these states are indexed by complex numbers $\xi=p+i q$ and, if $|\xi\rangle\langle\xi|$ is the projector onto the coherent state $|\xi\rangle$, we have that

$$
\begin{aligned}
& \int|\xi\rangle\langle\xi| d \xi d \xi^{*}=I \\
& \int \xi|\xi\rangle\langle\xi| d \xi d \xi^{*}=a
\end{aligned}
$$

and

$$
\int\left(|\xi|^{2}-1\right)|\xi\rangle\langle\xi| d \xi d \xi^{*}=a^{*} a .
$$

It is the extra term -1 in the last integral that kills the lower bound and gives an unwanted energy - 1 for each phonon mode (and hence a negative contribution to the energy that is infinite both because there is no infrared and no ultraviolet cutoff). In other 
words, coherent states would give us what we want (effectively replacing the operator $a$ by the number $\xi$ ), were it not for the unfortunate fact that the positive operator $a^{*} a$ is represented by the nonpositive symbol, $|\xi|^{2}-1$.

Our remedy will be to reduce the effective number of phonon modes to a finite number of $O\left(\alpha^{9 / 5}\right)$, independent of $V$. This will not sacrifice rigor because we shall prove that the reduction affects the energy only to $O\left(\alpha^{9 / 5}\right)$ at most. These modes will be quite different from the original $a_{k}$ modes; indeed they will be the $a_{k}$ modes summed over boxes in $\mathbf{k}$-space of size $\alpha^{3 / 5}$. Thus, our physical description of strong coupling will be a little different from the conventional one. Instead of saying that the phonons cannot follow the electron, our point of view will be that the electron significantly excites only finitely many field modes.

This mode reduction is accomplished in several steps which can be outlined as follows.

- I. Using a simple commutator estimate (as in Schrödinger's elementary proof of Heisenberg's uncertainty principle [22]) we can show that $|\mathbf{k}|$ values larger than $K \approx \alpha^{6 / 5}$ can be ignored with an energy cost of only $\alpha^{9 / 5}$. This eliminates the ultraviolet problem, i.e., the fact that $|\mathbf{k}|^{-2}$ is not summable. $\alpha^{-9 / 10}$

- II. With the same energy error we can localize the electron to a cube of side length

- III. We decompose the ball $|\mathbf{k}|<K$ into blocks of size $\alpha^{3 / 5}$, each containing $n \mathbf{k}$ values (with $n \sim V \alpha^{9 / 5}$ ). There are about $\alpha^{9 / 5}$ blocks. Because the electron coordinate $\mathbf{x}$ has been localized, the function $\exp (i \mathbf{k} \cdot \mathbf{x})$ in $H$ can be replaced, in a block $B$, by $\exp \left(i \mathbf{k}_{B} \cdot \mathbf{x}\right)$, where $\mathbf{k}_{B}$ is any conveniently chosen point in $B$; the energy penalty is again $\alpha^{9 / 5}$. The effect of this decoupling is that the electron interacts only with the one mode $A_{B} \approx n^{-1 / 2} \sum_{\mathbf{k} \in B} a_{\mathbf{k}}$, from block $B$. Thus, the electron ends up interacting with only $\alpha^{9 / 5}$ modes, which is a finite number!

- IV. These active modes can now be represented by coherent state integrals, as above. The unwanted -1 term now contributes only $\alpha^{9 / 5}$ to $E_{0}$, that being the number of modes.

The details will now be given.

I. We first establish a commutator inequality which will show that the large $\mathbf{k}$ modes of the Hamiltonian may be discarded at the price of only a small decrease in the coefficient of the $\mathbf{p}^{2}$ term of the Hamiltonian. For any normalized state with expectation $\langle\cdot\rangle$, we have

$$
\begin{array}{r}
\left|k_{j}\right|\left|\left\langle a_{\mathbf{k}} e^{i \mathbf{k} \cdot \mathbf{x}}\right\rangle\right|=\left|\left\langle\left[p_{j}, a_{\mathbf{k}} e^{i \mathbf{k} \cdot \mathbf{x}}\right]\right\rangle\right| \\
\leq 2\left\langle p_{j}^{2}\right\rangle^{1 / 2}\left\langle a_{\mathbf{k}}^{*} a_{\mathbf{k}}\right\rangle^{1 / 2}
\end{array}
$$

for each phonon momentum coordinate $k_{j}, j=1,2,3$, just by the Schwarz inequality. Squaring this inequality, summing over $j$, and then taking the square root, we obtain

$$
\left|\left\langle a_{\mathbf{k}} e^{i \mathbf{k} \cdot \mathbf{x}}\right\rangle\right| \leq \frac{2}{|\mathbf{k}|}\left\langle\mathbf{p}^{2}\right\rangle^{1 / 2}\left\langle a_{\mathbf{k}}^{*} a_{\mathbf{k}}\right\rangle^{1 / 2} .
$$

A similar inequality holds for $a_{\mathbf{k}}$ replaced by $a_{\mathbf{k}}^{*}$. It follows that for any $\varepsilon>0$, and with $K \equiv 8 \alpha / \pi \varepsilon$, 


$$
\begin{aligned}
& -\left(\frac{4 \pi \alpha}{V}\right)^{1 / 2} \sum_{|\mathbf{k}| \geq K}\left[\left\langle\frac{a_{\mathbf{k}}}{|\mathbf{k}|} e^{i \mathbf{k} \cdot \mathbf{x}}\right\rangle+c . c .\right] \\
& \leq 4\left(\frac{4 \pi \alpha}{V}\right)^{1 / 2}\left\langle\mathbf{p}^{2}\right\rangle^{1 / 2} \sum_{|\mathbf{k}| \geq K}|\mathbf{k}|^{-2}\left\langle a_{\mathbf{k}}^{*} a_{\mathbf{k}}\right\rangle^{1 / 2} \\
& \leq 4\left(\frac{4 \pi \alpha}{V}\right)^{1 / 2}\left\langle\mathbf{p}^{2}\right\rangle^{1 / 2}\left(\sum_{|\mathbf{k}| \geq K}|\mathbf{k}|^{-4}\right)^{1 / 2}\left(\sum_{|\mathbf{k}| \geq K}\left\langle a_{\mathbf{k}}^{*} a_{\mathbf{k}}\right\rangle\right)^{1 / 2} \\
& =2 \varepsilon^{1 / 2}\left\langle\mathbf{p}^{2}\right\rangle^{1 / 2}\left(\sum_{|\mathbf{k}| \geq K}\left\langle a_{\mathbf{k}}^{*} a_{\mathbf{k}}\right\rangle\right)^{1 / 2} \\
& \leq \varepsilon\left\langle\mathbf{p}^{2}\right\rangle+\sum_{|\mathbf{k}| \geq K}\left\langle a_{\mathbf{k}}^{*} a_{\mathbf{k}}\right\rangle
\end{aligned}
$$

again by the Schwarz inequality. (We have taken the limit $V \rightarrow \infty$ in the sum.)

The above inequality (10) is an ultraviolet bound. It shows that the Hamiltonian $H$ is bounded below by a new one $H_{K}$, i.e., $H \geq H_{K}$, with

$$
H_{K} \equiv\left(1-\frac{8 \alpha}{\pi K}\right) \mathbf{p}^{2}+\sum_{|\mathbf{k}|<K} a_{\mathbf{k}}^{*} a_{\mathbf{k}}+\left(\frac{4 \pi \alpha}{V}\right)^{1 / 2} \sum_{|\mathbf{k}|<K}\left[\frac{a_{\mathbf{k}}}{|\mathbf{k}|} e^{i \mathbf{k} \cdot \mathbf{x}}+h . c .\right]
$$

By completing the square, we have that

$$
a_{\mathbf{k}}^{*} a_{\mathbf{k}}+\left(\frac{4 \pi \alpha}{V}\right)^{1 / 2}\left[\frac{a_{\mathbf{k}}}{|\mathbf{k}|} e^{i \mathbf{k} \cdot \mathbf{x}}+\text { h.c. }\right] \geq-\frac{4 \pi \alpha}{|\mathbf{k}|^{2} V}
$$

and if $K$ is chosen so that the coefficient of $\mathbf{p}^{2}$ vanishes in $H_{K}$, i.e., $K=8 \alpha / \pi$, then

$$
H \geq H_{K} \geq-\frac{4 \pi \alpha}{V} \sum_{|\mathbf{k}|<K}|\mathbf{k}|^{-2}=-\frac{16}{\pi^{2}} \alpha^{2}
$$

showing that $H$ is indeed bounded below by $O\left(\alpha^{2}\right)$. But to obtain the sharp lower bound, we will take a much larger $K$, namely $K=\left(8 / \pi c_{1}\right) \alpha^{6 / 5}$. Here and below, the $c_{i}$ 's are constants independent of $\alpha$.

II. The next step is to localize the electron. Although a-priori the electron is in the box of volume $\mathrm{V}$, it actually can be confined to a box of much smaller size, with only a slight relative increase in its energy. More precisely, we have the following: Let $\Psi$ be any normalized state of the electron and phonons and define $E \equiv\left\langle\Psi\left|H_{K}\right| \Psi\right\rangle$. Then, given $\Delta E>0$, there exists a function $\phi$ of the electron coordinate $\mathbf{x}$ alone (but depending on $\Psi)$, with support in some cube of side length $L=\pi(3 / \Delta E)^{1 / 2}$ such that

$$
\left\langle\phi \Psi\left|H_{K}\right| \phi \Psi\right\rangle /\langle\phi \Psi \mid \phi \Psi\rangle \leq E+\Delta E
$$

To see this, let $\phi(\mathbf{x})=\prod_{j=1}^{3} \cos \left((\Delta E / 3)^{1 / 2} x_{j}\right)$ inside the cube of side length $\pi(3 / \Delta E)^{1 / 2}$ centered at the origin and $\phi=0$ outside the cube. Let $\phi_{y}(x)=\phi(x-y)$ and consider the integral 


$$
\begin{aligned}
& \int\left(\left\langle\phi_{\mathbf{y}} \Psi\left|H_{K}\right| \phi_{\mathbf{y}} \Psi\right\rangle-(E+\Delta E)\left\langle\phi_{\mathbf{y}} \Psi \mid \phi_{\mathbf{y}} \Psi\right\rangle\right) d^{3} y \\
& =\int\left(|\nabla \phi|^{2}-\Delta E|\phi|^{2}\right) d^{3} y=0 .
\end{aligned}
$$

(Note here that the cross terms $\nabla \phi_{\mathbf{y}} \nabla \Psi$ vanish after the $y$-integration.) Evidently, there must be a point $y$ such that at this point, the integrand on the left side of (15) is nonpositive (and where $\left\langle\phi_{\mathbf{y}} \Psi \mid \phi_{\mathbf{y}} \Psi\right\rangle$ is non-zero). This $\phi_{\mathrm{y}}$ is the $\phi$ that we need. The electron localization is now complete.

For our purposes, we take $\Delta E=c_{2} \alpha^{9 / 5}$, and the assertion above together with the ultraviolet bound then implies that the ground state energy $E_{0}$ of $H$ satisfies

$$
E_{0} \geq \inf _{\Psi}^{\prime}\left\langle\Psi\left|H_{K}\right| \Psi\right\rangle-c_{2} \alpha^{9 / 5}
$$

where the infimum is taken over all normalized $\Psi$ 's but having their $\mathrm{x}$-support in a cube of side length no larger than $L=\left(3 / c_{2}\right)^{1 / 2} \pi \alpha^{-9 / 10}$ somewhere in the large volume $V$.

III. The next step is to group the phonon modes together into blocks, which we take to be cubes with sides of length $P=c_{3} \alpha^{3 / 5}$ or, more precisely, the portion of those cubes lying in the big ball $\{\mathbf{k}:|\mathbf{k}|<K\}$. Let $B$ be a block (cube) of momenta with sides of length $P=c_{3} \alpha^{3 / 5}$ and let $\mathbf{k}_{B}$ be any fuxed point within this block whose precise position will be determined later. It is clear that the set of momenta $\{\mathbf{k}:|\mathbf{k}|<K\}$ can be covered by $N \equiv \frac{4}{3} \pi K^{3} / P^{3}+$ lower order $=\left(2^{11} /\left(3 \pi^{2} c_{1}^{3} c_{3}^{3}\right)\right) \alpha^{9 / 5}+$ lower order such blocks.

Assume that $\mathbf{x}$ itself varies over a cube of side length no bigger than $L$ with center at a point $x_{0}$ which we take to be the origin 0 . (If $x_{0}$ is not the origin, phase factors $\exp \left( \pm i\left(\mathbf{k}-\mathbf{k}_{B}\right) \cdot \mathbf{x}_{0}\right)$ standing before $a_{\mathbf{k}}$ and $a_{\mathbf{k}}^{*}$ in the Hamiltonian $H_{K}$ are readily eliminated by a unitary transformation involving the phonon variables only.) We have that for $\mathbf{k} \in B$,

$$
\left|e^{i \mathbf{k} \cdot \mathbf{x}}-e^{i \mathbf{k}_{B} \cdot \mathbf{x}}\right| \leq\left|\left(\mathbf{k}-\mathbf{k}_{B}\right) \cdot \mathbf{x}\right| \leq \frac{3 \pi c_{3}}{2}\left(\frac{3}{c_{2}}\right)^{1 / 2} \alpha^{-3 / 10}
$$

Then, for any $\delta>0$, we have the following inequality, which is obtained by completing the square, as in (12),

$$
\begin{aligned}
\sum_{B} \sum_{\mathbf{k} \in B}\left[\delta a_{\mathbf{k}}^{*} a_{\mathbf{k}}+\left(\frac{4 \pi \alpha}{V}\right)^{1 / 2}\left[\frac{a_{\mathbf{k}}}{|\mathbf{k}|}\left(e^{i \mathbf{k} \cdot \mathbf{x}}-e^{i \mathbf{k}_{B} \cdot \mathbf{x}}\right)+h . c .\right]\right] \\
\geq-\frac{4 \pi \alpha}{\delta V}\left(\frac{3 \pi c_{3}}{2}\left(\frac{3}{c_{2}}\right)^{1 / 2} \alpha^{-3 / 10}\right)^{2} \sum_{|\mathbf{k}|<K}|\mathbf{k}|^{-2} \\
=-\frac{108 c_{3}^{2}}{c_{1} c_{2} \delta} \alpha^{8 / 5}
\end{aligned}
$$

We make the choice $\delta=c_{4} \alpha^{-1 / 5}$.

The above inequality (19) permits us to bound $H_{K}$ (still restricted to states with $\mathbf{x}$-support in a cube of sides with size $L$ ) from below in the following way: 


$$
\begin{aligned}
H_{K} & \geq\left(1-c_{1} \alpha^{-1 / 5}\right) \mathbf{p}^{2}+\sum_{B} \sum_{\mathbf{k} \in B}\left[\left(1-c_{4} \alpha^{-1 / 5}\right) a_{\mathbf{k}}^{*} a_{\mathbf{k}}\right. \\
& \left.+\left(\frac{4 \pi \alpha}{V}\right)^{1 / 2}\left(\frac{a_{\mathbf{k}}}{|\mathbf{k}|} e^{i \mathbf{k}_{B} \cdot \mathbf{x}}+\text { h.c. }\right)\right]-\frac{108 c_{3}^{2}}{c_{1} c_{2} c_{4}} \alpha^{9 / 5}
\end{aligned}
$$

Note that, within a single block, the exponential factors multiplying the $a_{\mathbf{k}}$ - and $a_{\mathbf{k}}^{*}$-terms are now independent of $\mathbf{k}$. With this in mind, we define, for each block $B$, a block annihilation operator

$$
A_{B} \equiv\left(\sum_{\mathbf{k} \in B}|\mathbf{k}|^{-2}\right)^{-1 / 2} \sum_{\mathbf{k} \in B} \frac{a_{\mathbf{k}}}{|\mathbf{k}|}
$$

and its corresponding adjoint $A_{B}^{*}$. These operators are properly normalized boson modes, i.e.,

$$
\left[A_{B}, A_{B^{\prime}}^{*}\right]=\delta_{B, B^{\prime}}
$$

Then

$$
\sum_{\mathbf{k} \in B} a_{\mathbf{k}}^{*} a_{\mathbf{k}} \geq A_{B}^{*} A_{B}
$$

and so the operator terms on the right hand side of (19) exceed the operator $H_{K}^{b l o c k} \equiv$ $H_{K}^{\text {block }}\left(\left\{\mathbf{k}_{B}\right\}\right)$ defined by

$$
\begin{aligned}
H_{K}^{\text {block }} \equiv & \left(1-c_{1} \alpha^{-1 / 5}\right) \mathbf{p}^{2}+\sum_{B}\left[\left(1-c_{4} \alpha^{-1 / 5}\right) A_{B}^{*} A_{B}\right. \\
& \left.+\left(\frac{4 \pi \alpha}{V} \sum_{\mathbf{k} \in B}|\mathbf{k}|^{-2}\right)^{1 / 2}\left(A_{B} e^{i \mathbf{k}_{B} \cdot \mathbf{x}}+\text { h.c. }\right)\right]
\end{aligned}
$$

Referring to (16) and (19), we can summarize the situation: The ground state energy $E_{0}$ of $H$ satisfies

$$
E_{0} \geq \inf _{\Psi} \sup _{\left\{\mathbf{k}_{B}\right\}}\left\langle\Psi\left|H_{K}^{\text {block }}\right| \Psi\right\rangle-\left(c_{2}+\frac{108 c_{3}^{2}}{c_{1} c_{2} c_{4}}\right) \alpha^{9 / 5}
$$

where the infimum can now even be taken over $\Psi$ 's with no restriction on their $\mathbf{x}$-support, and with the $\mathbf{k}_{B}$ 's chosen optimally, depending on $\Psi$.

IV. In order to get a lower bound on $H_{K}^{\text {block }}$, we use the technology of coherent states. Let $|\xi\rangle=\pi^{-1 / 2} \exp \left(-\frac{1}{2}|\xi|^{2}+\xi A^{*}\right)|0\rangle$ denote a normalized coherent state for a single harmonic oscillator. In terms of these states one has the identity

$$
\exp (\mu A) \exp \left(\nu A^{*}\right)=\int \exp \left(\mu \xi+\nu \xi^{*}\right)|\xi\rangle\langle\xi| d \xi d \xi^{*},
$$

from which the identities for the operators $A$ and $A^{*} A$ mentioned in the introduction are readily obtained.

Let $\Psi$ be any normalized state and let $|\xi\rangle=\prod_{B}\left|\xi_{B}\right\rangle$ denote a tensor product of coherent states of the block modes corresponding to the operators $\left\{A_{B}\right\}$. Set $\Psi_{\xi}(\mathbf{x})=$ $\langle\xi \mid \Psi\rangle_{\text {phonon }}$, where the inner product, $\langle\mid\rangle_{\text {phonon }}$, is just over the phonon variables, not x. Then 


$$
\left\langle\Psi\left|H_{K}^{\text {block }}\right| \Psi\right\rangle=\int\left\langle\Psi_{\xi}\left|h_{\xi}\right| \Psi_{\xi}\right\rangle_{\text {electron }} \prod_{B} d \xi_{B} d \xi_{B}^{*},
$$

where the inner product in the integrand, $\langle\mid\rangle_{\text {electron }}$, is over the electronic coordinates $\mathbf{x}$, and $h_{\xi}$ is the Schrödinger operator

$$
\begin{aligned}
h_{\xi} & \equiv\left(1-c_{1} \alpha^{-1 / 5}\right) \mathbf{p}^{2} \\
& +\sum_{B}\left[\left(1-c_{4} \alpha^{-1 / 5}\right)\left(\left|\xi_{B}\right|^{2}-1\right)+\left(\frac{4 \pi \alpha}{V} \sum_{\mathbf{k} \in B}|\mathbf{k}|^{-2}\right)^{1 / 2}\left(\xi_{B} e^{i \mathbf{k}_{B} \cdot \mathbf{x}}+\text { c.c. }\right)\right] .
\end{aligned}
$$

Now the supremum over $\mathbf{k}_{B}$ of (26) exceeds what is obtained by completing the square, namely,

$$
\begin{aligned}
& \sup _{\left\{\mathbf{k}_{B}\right\}} \int\left[\left(1-c_{1} \alpha^{-1 / 5}\right)\left\langle\Psi_{\xi}\left|\mathbf{p}^{2}\right| \Psi_{\xi}\right\rangle_{\text {electron }}\right. \\
& \left.-\frac{4 \pi \alpha}{\left(1-c_{4} \alpha^{-1 / 5}\right) V} \sum_{B} \sum_{\mathbf{k} \in B} \frac{\left|\hat{\rho}_{\xi}\left(\mathbf{k}_{B}\right)\right|^{2}}{|\mathbf{k}|^{2} \hat{\rho}_{\xi}(\mathbf{0})}\right] \prod_{B} d \xi_{B} d \xi_{B}^{*}-N,
\end{aligned}
$$

where $\hat{\rho}_{\xi}(\mathbf{k})=\left\langle\Psi_{\xi}|\exp (i \mathbf{k} \cdot \mathbf{x})| \Psi_{\xi}\right\rangle_{\text {electron }}$ is the Fourier transform of $\left|\Psi_{\xi}\right|^{2}(\mathbf{x})$. (Here, we can remove the $|\mathbf{k}|<K$ restriction on the $\mathbf{k}$-sum.) We emphasize again that were it not for the grouping of the phonons into blocks, then this coherent state estimate would contain a negative constant equal to the number of phonon modes with $|\mathbf{k}|<K$, which is infinite in the $V \rightarrow \infty$ limit, instead of $N=O\left(\alpha^{9 / 5}\right)$.

At this point we choose $k_{B}$ to be a point in $B$ where the function of $\mathbf{k}$ given by

$$
\int \frac{\left|\hat{\rho}_{\xi}(\mathbf{k})\right|^{2}}{\hat{\rho}_{\xi}(\mathbf{0})} \prod_{B} d \xi_{B} d \xi_{B}^{*}
$$

is minimal. This point depends on $\Psi$. With this choice, (28) is, in turn, larger than

$$
\begin{aligned}
& \int\left[\left(1-c_{1} \alpha^{-1 / 5}\right)\left\langle\Psi_{\xi}\left|\mathbf{p}^{2}\right| \Psi_{\xi}\right\rangle_{\text {electron }}\right. \\
& \left.-\frac{4 \pi \alpha}{\left(1-c_{4} \alpha^{-1 / 5}\right) V} \sum_{\mathbf{k}} \frac{\left|\hat{\rho}_{\xi}(\mathbf{k})\right|^{2}}{|\mathbf{k}|^{2} \hat{\rho}_{\xi}(\mathbf{0})}\right] \prod_{B} d \xi_{B} d \xi_{B}^{*}-N .
\end{aligned}
$$

But the integrand of this integral is $\left\langle\Psi_{\xi} \mid \Psi_{\xi}\right\rangle_{\text {electron }} \times \mathcal{E}\left(\Psi_{\xi} /\left(\left\langle\Psi_{\xi} \mid \Psi_{\xi}\right\rangle_{\text {electron }}^{1 / 2}\right)\right)$, where $\mathcal{E}(\cdot)$ is just the Pekar functional (2), (but with coefficients altered by $O\left(\alpha^{-1 / 5}\right)$ ); the ksum is the Fourier series for the Coulomb self-energy term of $\mathcal{E}$. Thus, the integral (30) exceeds $\left(1-c_{1} \alpha^{-1 / 5}\right)^{-1}\left(1-c_{4} \alpha^{-1 / 5}\right)^{-2} E_{P}(\alpha)$, with $E_{P}(\alpha)$ being the Pekar minimum energy of (2).

Taking into account the altered coefficients in the Pekar functional, the lower bound (24), and the definition of $N$, we obtain

$$
E_{0}(\alpha) \geq E_{P}(\alpha)-\left(c_{2}+\frac{108 c_{3}^{2}}{c_{1} c_{2} c_{4}}+\frac{2^{11}}{3 \pi^{2} c_{1}^{3} c_{3}^{3}}+c_{5}\right) \alpha^{9 / 5}-o\left(\alpha^{9 / 5}\right),
$$

where $E_{P}(\alpha)=-c_{P} \alpha^{2}, c_{P}=0.109,[11]$, and where $c_{5}=\left(c_{1}+2 c_{4}\right) c_{P}$ accounts for the altered coefficients in the variational principle. This completes the proof of the lower bound. Optimizing the coefficients, we find that the $\alpha^{9 / 5}$-error term in (31) is no greater than $3.822 \alpha^{9 / 5}$. 


\section{References}

1. Fröhlich, H.: Proc. Phys. Soc. A160, 230 (1937); Electrons in lattice fields. Adv. in Phys. 3, 325-361 (1954)

2. Pekar, S.I.: Untersuchung über die Elektronentheorie der Kristalle. Berlin: Akademie Verlag, 1954

3. Bogolyubov, N.N.: On a new form of the adiabatic theory of disturbances in the problem of interaction of particles with a quantum field (Russian). Ukr. Mat. Zh 2, 3-24 (1950)

4. Tyablikov, S.V.: An adiabatic form of perturbation theory in the problem of the interaction of a particle with a quantum field (Russian). Zh. Eksper. Teor. Fiz. 21, 377-388(1951)

5. Gross, E.P.: Small oscillation theory of the interaction of a prticle in a scalar field. Phys. Rev. 100, 1571-1578 (1955); Strong coupling polaron theory and translation invariance. Ann. Phys. (NY) 99, 1-29(1976)

6. Fröhlich, J.: On the infrared problem in a model of scalar electrons and massless scalar bosons. Ann. Inst. Poincare A 19, 1-103; Existence of dressed one electron states in a class of persistent models. Fortschr. Phys. 22, 159-198 (1974)

7. Spohn, H.: Roughening and pinning transition for the polaron, J. Phys. A 19, 533-545(1986); Effective mass of the polaron: A functional integral approach. Ann. Phys. (NY), 175, 278-318(1987); The polaron at large total momentum. J. Phys. A 21, 1199-1211 (1988)

8. Lee, T.D., Low, F. and Pines, D.: The motion of slow electrons in a polar crystal. Phys. Rev. 90, 297-302 (1953)

9. Schultz, T.D.: "Electron-Lattice Interactions in Polar crystals." Technical Report no. 9 Solid State and Molecular Theory Group, M.I.T. , August 20, (1956). See also article in Polarons and Excitons, Scottish Summer School (1962), eds. C.G. Kuper and G.D. Whitfield, Edinburgh, London: Oliver and Boyd, 1963

10. Landau, L.D.: Electron motion in crystal lattices. Phys. Z. Sowjet 3, 664-665 (1933)

11. Gerlach, B. and Löwen, H.: Analytical properties of polaron systems or: Do polaronic phase transitions exist or not? Rev. Mod. Phys. 63, 63-90 (1991)

12. Peeters, F. M. and Devreese, J. T.: On the existence of a phase transition for the Fröhlich polaron. Phys. Status Solidi B 112, 219-229 (1982)

13. Lieb, E.H.: Existence and uniqueness of the minimizing solution of Choquard's nonlinear equation. Stud. Appl. Math. 57, 93-105(1977)

14. Lieb, E.H. and Yamazaki, K.: Ground-state energy and effective mass of the polaron. Phys. Rev. 111, 728-733 (1958)

15. Adamowski, J.: Gerlach, B. and Leschke, H.: Strong-coupling limit of polaron energy, revisited. Phys. Lett. 79A, 249-251 (1980)

16. Donsker, M. and Varadhan, S.R.S.: Asymptotics for the polaron. Commun. Pure Appl. Math. 36, 505-528 (1983); Asymptotic evaluation of certain Markov process expectations for large times, IV. Comm. Pure Appl. Math. 36, 183-212 (1983)

17. Varadhan, S.R.S.: Private communication

18. Feynman, R.P.: Slow electrons in a polar crystal. Phys. Rev. 97, 660-665 (1955)

19. Ginibre, J.: Applications of functional integration. In: Statistical Mechanics and Quantum Field Theory, C. DeWitt and R. Stora eds., London-New York: Gordon and Breach, 1971, pp. 327-427. See pages 420-426

20. Roepstorff, G.: Path integral approach to quantum physics. Berlin-Heidelberg-New York: Springer, 1994. See Sect. 5.3

21. Fischer, W., Leschke, H. and Müller, P.: In Path Integrals in Physics, Bangkok, 1993, eds. V. Sa-yakanit, J.O. Berananda and W. Sritrakool, Singapore: World Scientific, 1994, pp. 347-354

22. Schrödinger, E.: Zum Heisenbergschen Unschärfe Prinzip, Sitzungsber. der Preuss. Akad. der Wiss., Berlin: Phys-Math Klasse XIX, 1930, pp.348-356 
